\title{
Shooting two birds with two bullets: how to find Minimum Mean OSPA estimates
}

\author{
Marco Guerriero ${ }^{\dagger}$, Lennart Svensson ${ }^{\ddagger}$, Daniel Svensson $^{\ddagger}$, \\ Peter Willett*
}

\author{
${ }^{\dagger}$ Research \& Advanced System Design Department, Elettronica S.p.A., Italy \\ $\ddagger$ Department of Signals and Systems, Chalmers University of Technology, Gothenburg, Sweden \\ *Dept. of Electr. and Comp. Engineering, University of Connecticut, USA*
}

\begin{abstract}
Most area-defense formulations follow from the assumption that threats must first be identified and then neutralized. This is reasonable, but inherent to it is a process of labeling: threat $A$ must be identified and then threat $B$, and then action must be taken. This manuscript begins from the assumption that such labeling $(A \& B)$ is irrelevant. The problem naturally devolves to one of Random Finite Set (RFS) estimation: we show that by eschewing any concern of target label we relax the estimation procedure, and it is perhaps not surprising that by such a removal of constraint (of labeling) performance (in terms of localization) is enhanced. A suitable measure for the estimation of unlabeled objects is the Mean OSPA (MOSPA). We derive a general algorithm which provided the optimal estimator which minimize the MOSPA. We call such an estimator a Minimum MOSPA (MMOSPA) estimator.
\end{abstract}

Keywords: MSE, OSPA, Miss-distance, target tracking.

\section{Introduction}

The Mean Squared Error (MSE) has long been the dominant quantitative performance metric in the field of signal processing. For the traditional multi-target tracking algorithms, the aim is to minimize the MSE between target truths and the corresponding track estimates. The MSE is the nearly ubiquitous preference of design engineers seeking to optimize signal processing algorithms. For example, in the Joint Probabilistic Data Association (JPDA) filter [5], the Minimum MSE (MMSE) — the posterior mean - of the state of the targets can be written in a nice recursive way.

For the problem of tracking the unordered set of targets, that is when the target labels are irrelevant, such a measure is not suitable, so another metric is required. In this article, we propose to use the Optimal Subpattern Assignment (OSPA) metric [3]. To describe the

\footnotetext{
*Marco Guerriero and Peter Willett were partially supported by the ONR under contracts N00014-09-10613 and N00014-1010412 .
}

problem of tracking an unordered set of targets, a Random Finite Set (RFS) description of the target states is suitable. An RFS, which is a part of the Finite Set Statistics (FISST) framework [4], is a set with a random number of elements and where each element is a random entity. Two popular algorithms, namely the Probability Hypothesis Density (PHD) and the Cardinalized PHD (CPHD) filters [6-9], have been derived in the FISST framework.

The MOSPA metric has been recently used as a measure to evaluate the performance of some multitarget tracking algorithms. But the following question arises: How should we (and, indeed, should we) design a new class of estimators oriented to the MOSPA? This question is the concern of this article. To the best of our knowledge, theoretical results on how to obtain Minimum Mean OSPA (MMOSPA) estimates are still missing.

The SJPDA filter [10] is an adjusted version of the JPDA filter, designed to perform well according to the OSPA measure. The SJPDA exploits the fact that many ordered densities are equivalent from an RFS perspective. The set of densities which are equivalent is called an RFS family and the idea in the SJPDA is find a density in the family which is approximately Gaussian. In a related fashion, the MMOSPA estimates desired here are obtained as the mean of a particular density within the same family. To simplify the presentation we limit our discussions to static problems, but we hope to report results for dynamic situations soon.

In this paper we derive a general algorithm which provides the MMOSPA estimates for an arbitrary, but known, number of targets. In particular we will give an explicit algorithm for the two target case, here cast as the problem of a hunter shooting birds. In Figure 1 two unfortunate birds are depicted and the aim of the hunter is to shoot both birds without regard for which is struck first and which second. To evaluate the quality of the hunter's ability we pick a measure that captures the hunter's chances to shoot both birds. In 


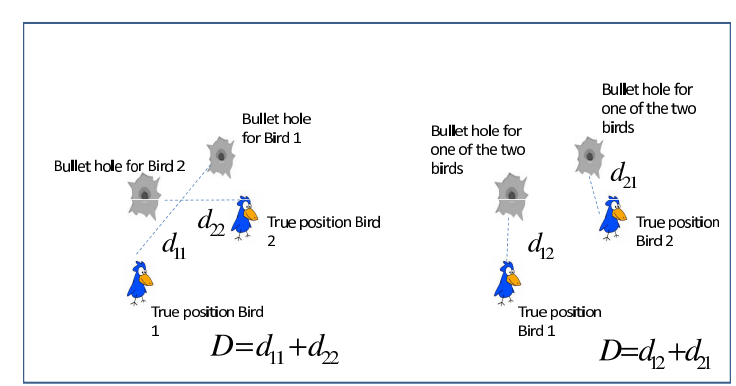

Figure 1: Shooting two birds with two bullets.

the left area of Figure 1, the MMSE estimates of the two birds locations are sought to minimize the squared error (SE), whereas in the right area of Figure 1 the two unlabeled estimates (MMOSPA estimates) of the two birds locations are are sought via a metric that ignores bird labeling, i.e., the MOSPA. We remark the fact that in the second case, in order to have a smaller metric $D$, we have to relax the problem by discarding the identity of the birds estimates.

The remainder of the paper is organized as follows. In Section 2 we formalize our problem and provide some of motivations for it and in Section 3 we briefly introduce the concept of ordered densities. Section 4 contains our main theoretical contributions and also provides a general algorithm to find the MMOSPA estimates. Our theoretical findings are validated through simulated examples in Section 5 and, finally, in Section 6 we summarize.

\section{Problem Formulation}

In this article we study the problem of static estimation of an arbitrary, but known, number of targets without concern regarding their identities. We introduce the random set of targets

$$
\mathbf{X}=\left\{\mathbf{x}^{(1)}, \mathbf{x}^{(2)}, \ldots, \mathbf{x}^{(n)}\right\}
$$

where $\mathbf{x}^{(i)}$ is the state vector of target number $i$, which we assume does not include a target identity label, and where $n$ is the number of targets in the scene. What we seek is an estimate $\widehat{\mathbf{X}}$ of the set of targets which minimizes the MOSPA (Mean OSPA) measure, defined later. We call such an estimate a Minimum MOSPA (MMOSPA) estimate.

Now, a common measure in the literature is the squared error (SE)

$$
\begin{aligned}
\operatorname{SE}\left(\hat{\mathbf{X}},\left[\mathbf{x}^{(1)}, \ldots, \mathbf{x}^{(n)}\right]\right) \\
\quad=\frac{1}{n}\left(\left(\hat{\mathbf{x}}^{(1)}-\mathbf{x}^{(1)}\right)^{2}+\cdots+\left(\hat{\mathbf{x}}^{(n)}-\mathbf{x}^{(n)}\right)^{2}\right)
\end{aligned}
$$

To evaluate the measure, we need estimates of the states of target 1, 2, and so on, i.e., of the labeled targets.
Since this paper is about describing where there are targets, rather than where a target with a certain label is, the squared error is not a good measure. We note for example in the two-bird case, that if the identities of the birds have been exchanged, the squared error can be very large, even though there might be two accurate estimates available, i.e., even though $\hat{\mathbf{x}}^{(1)} \approx \mathbf{x}^{(2)}$ and $\hat{\mathbf{x}}^{(2)} \approx \mathbf{x}^{(1)}$. Clearly, the SE is not a good choice since we seek a measure that can capture the quality of estimates of the set of birds (targets).

Several multi-target performance measures have been proposed in the literature. The first attempt to a multitarget performance measure was given in [1]. There, an optimal assignment is performed to find the best association of state estimates and true-target states. The cost function is arbitrary, and could for example be the squared distance. The attempt is creative and original, but ultimately suffers from the lack of mathematical underpinnings available at the time.

The first rigorous theory of multi-object distances was given in [2]. The measure, which is based on the optimal assignment approach, is called the Optimal Mass Transfer (OMAT) metric. When the number of targets is known, the OMAT metric is identical to the optimal assignment procedure. The OMAT has a number of weaknesses, discussed in [3]. The major weaknesses appear when the number of targets is not known and the estimated number is not always equal to the true number. To remedy the shortcomings, a new metric, called the Optimal Subpattern Assignment (OSPA) metric, was proposed in [3]. Since OSPA is an intuitively appealing measure, and has received much attention of late, we are using it as the basis measure for the problem. As the number of targets is assumed known, we will only present OSPA for a known number of targets; for a full description, see [3].

Let $\mathbf{X}$ be the set of true target states and $\widehat{\mathbf{X}}$ be the set of target estimates, both with $n$ elements. The OSPA measure $\tilde{d}_{p}^{(c)}$ is then defined as

$$
\tilde{d}_{p}^{(c)}(\widehat{\mathbf{X}}, \mathbf{X})=\left(\frac{1}{n}\left(\min _{\pi \in \Pi_{n}} \sum_{i=1}^{n} d^{(c)}\left(\mathbf{x}^{(i)}, \hat{\mathbf{x}}^{\pi(i)}\right)^{p}\right)\right)^{1 / p}
$$

Here, $d^{(c)}(\mathbf{x}, \hat{\mathbf{x}}) \triangleq \min (c, d(\mathbf{x}, \hat{\mathbf{x}}))$ is the distance $d$ between $\mathbf{x}$ and $\hat{\mathbf{x}}$, cut-off at $c$. Further, $\Pi_{n}$ is the set of all possible permutations of $\widehat{\mathbf{X}}$. The notation $\hat{\mathbf{x}}^{\pi(i)}$ describes the $i^{\text {th }}$ permutation (re-ordering) of the vector $\hat{\mathbf{x}}$. In this article, we let $d$ be the Euclidean distance, we use a quadratic measure $(p=2)$ and $c=\infty$, (the number of estimated targets is equal to the number of true targets). Through the minimization, the measure performs an optimal assignment of target estimates to true target states, possibly cut-off at $c$. A consequence of knowing the number of targets and using $c=\infty$ is that the OSPA, OMAT and optimal assignment approach all reduce to the same thing. 
To obtain a measure for which we can define an optimal multi-object estimator, we average over the density $p(\mathbf{X})$ which gives us a definition of the mean OSPA (MOSPA) measure

$$
\operatorname{MOSPA}_{p}^{(c)}(\widehat{\mathbf{X}}, p(\mathbf{X})) \triangleq \mathbb{E}_{p(\mathbf{X})}\left\{\tilde{d}_{p}^{(c)}\right\} .
$$

The density $p(\mathbf{X})$ should be thought of as the posterior density $^{1}$. Throughout the paper we will use the notation $\operatorname{MOSPA}(\widehat{\mathbf{X}}, p(\mathbf{X}))$ to denote the MOSPA metric. An optimal estimator, in the MOSPA sense, is an estimator which minimizes the MOSPA measure. Such an estimator is referred to as a minimum MOSPA (MMOSPA) estimator ${ }^{2}$.

\subsection{Motivation of the problem}

The problem that we study in this paper is that of estimating an unordered set of targets, for which the MMOSPA estimator is optimal (in the MOSPA sense). There are many problems where the ordering of the targets is not of interest. That is, to solve those problems, it is not required to estimate the identities of the targets, but only to estimate the set of targets. We here give some examples of when minimizing MOSPA provides a solution which is more reasonable than what is obtained when minimizing the Mean Square Error (MSE), using an ordered density.

A first application when MOSPA is reasonable is in radar cueing, i.e., for the problem of steering a radar sensor to areas of high target existence probability. In such cases, there is no interest in which target is which, but the question of where there are targets is very important. Another example is found in the automotive industry: in collision avoidance systems, it is not of interest to know which car is which, the goal is to avoid all cars. As a further example: in some NATO maritime surveillance actions it is important to send helicopters as close as possible to a set of vessels with anomalous behavior without concern of knowing which anomalous vessel is which.

\section{Ordered densities}

An RFS, which is part of the FISST framework [4], is a set with a random number of elements and where each element is a random quantity. Here the only aspect to FISST that is key to our findings is given by the relation between an RFS density and an ordered density. For $n$ targets, the relation between an RFS density $f\left(\left\{\mathbf{x}_{1}, \ldots, \mathbf{x}_{n}\right\}\right)$ and an ordered density $p\left(\mathbf{x}_{1}, \ldots, \mathbf{x}_{n}\right)$ is

$$
f\left(\left\{\mathbf{x}_{1}, \ldots, \mathbf{x}_{n}\right\}\right)=\sum_{i=1}^{n !} p\left(\pi_{i}\left(\mathbf{x}_{1}, \ldots, \mathbf{x}_{n}\right)\right) .
$$

\footnotetext{
${ }^{1}$ Any practical setting would involve measurements. If we denote these by $\mathbf{Z}, p(\mathbf{X})$ should be replaced be $p(\mathbf{X} \mid \mathbf{Z})$. However, for notation we exclude $\mathbf{Z}$ from our presentation.

${ }^{2}$ Note that the relation between OSPA, MOSPA and MMOSPA is analogous to that between the familiar acronyms SE, MSE and MMSE.
}

With $\pi_{i}\left(\mathbf{x}_{1}, \ldots, \mathbf{x}_{n}\right)$ we denote the permutation of the index set $\{1, \ldots, n\}$ on the vector $\left(\mathbf{x}_{1}, \ldots, \mathbf{x}_{n}\right)$; for instance, for $n=2$ we use $\pi_{1}\left(\mathbf{x}_{1}, \mathbf{x}_{2}\right)=\left(\mathbf{x}_{1}, \mathbf{x}_{2}\right)$ and $\pi_{2}\left(\mathbf{x}_{1}, \mathbf{x}_{2}\right)=\left(\mathbf{x}_{2}, \mathbf{x}_{1}\right)$. Using (5), we can calculate the RFS density from an ordered density. One important consequence of this relation is described in the following proposition.

Proposition 1 For $n>1$, the mapping from densities of ordered state vectors, $p_{i}\left(\mathbf{x}_{1}, \ldots, \mathbf{x}_{n}\right)$, to RFS densities, $f\left(\left\{\mathbf{x}_{1}, \ldots, \mathbf{x}_{n}\right\}\right)$, is many-to-one.

The relevance of this proposition is related to the fact that all such densities should result in the same RFS state estimates.

Definition 1 When two labeled densities, $p_{1}\left(\mathbf{x}_{1}, \ldots, \mathbf{x}_{n}\right)$ and $p_{2}\left(\mathbf{x}_{1}, \ldots, \mathbf{x}_{n}\right)$, correspond to the same RFS density we say that they belong to the same RFS family.

That is, using (5), we obtain the same RFS density regardless if $p_{1}\left(\mathbf{x}_{1}, \ldots, \mathbf{x}_{n}\right)$ or $p_{2}\left(\mathbf{x}_{1}, \ldots, \mathbf{x}_{n}\right)$ is used in the calculation.

\section{Computing MMOSPA esti- mates}

In this section we present our main contributions. The key results from a theoretical perspective are summarized in Theorems 1 and 2 in Section 4.1. Details regarding how to evaluate the estimators are given in Section 4.2 and 4.3 .

\subsection{Relation between MOSPA and MSE}

In the multi-target state estimation the usual definition for the MSE is

$$
\operatorname{MSE}(\hat{\mathbf{x}}, p(\mathbf{x}))=\frac{1}{n} \int\left(\sum_{i=1}^{n} d\left(\hat{\mathbf{x}}_{i}, \mathbf{x}_{i}\right)\right) p(\mathbf{x}) d \mathbf{x}
$$

where the averaging is with respect to one particular ordered density. In an analogous way, we have that MOSPA, from (4), can be expressed as

$$
\operatorname{MOSPA}(\hat{\mathbf{x}}, p(\mathbf{x}))=\int \tilde{d}_{p}^{(c)}(\hat{\mathbf{x}}, \mathbf{x}) p(\mathbf{x}) d \mathbf{x}
$$

where the averaging is with respect to an ordered density $p(\mathbf{x})$ within the RFS family. The following lemma shows that all ordered densities within the same RFS family yield the same MOSPA.

Lemma 1 For any ordered density $p(\mathbf{x})$ within the same RFS family, it holds that $\operatorname{MOSPA}(\hat{\mathbf{x}}, p(\mathbf{x}))$ does not depend on the particular choice of $p(\mathbf{x})$. 
Proof of Lemma 1 By letting

$$
\mathcal{A}(\hat{\mathbf{x}})=\left\{\mathbf{x}: \frac{1}{n} \sum_{i=1}^{n} d\left(\hat{\mathbf{x}}_{i}, \mathbf{x}_{i}\right)=\tilde{d}_{p}^{(c)}(\hat{\mathbf{x}}, \mathbf{x})\right\}
$$

we have that:

$$
\begin{aligned}
\int \tilde{d}_{p}^{(c)}(\hat{\mathbf{x}}, \mathbf{x}) p(\mathbf{x}) d \mathbf{x} & =\int_{\mathcal{A}(\hat{\mathbf{x}})} \tilde{d}_{p}^{(c)}(\hat{\mathbf{x}}, \mathbf{x}) p(\mathbf{x}) d \mathbf{x} \\
& +\sum_{i=2}^{n !} \int_{\mathcal{A}(\hat{\mathbf{x}})} \tilde{d}_{p}^{(c)}(\hat{\mathbf{x}}, \mathbf{x}) p\left(\pi_{i}(\mathbf{x})\right) d \mathbf{x} \\
& =\int_{\mathcal{A}(\hat{\mathbf{x}})} \tilde{d}_{p}^{(c)}(\hat{\mathbf{x}}, \mathbf{x}) \sum_{i=1}^{n !} p\left(\pi_{i}(\mathbf{x})\right) d \mathbf{x} \\
& =\int_{\mathcal{A}(\hat{\mathbf{x}})} \tilde{d}_{p}^{(c)}(\hat{\mathbf{x}}, \mathbf{x}) f(\{\mathbf{x}\}) d \mathbf{x}
\end{aligned}
$$

which is the same for all ordered densities $p(\mathbf{x})$, within the same RFS family.

From the definitions of MSE and MOSPA, and from the fact that $\frac{1}{n} \sum_{i=1}^{n} d\left(\hat{\mathbf{x}}_{i}, \mathbf{x}_{i}\right) \geq \tilde{d}_{p}^{(c)}(\hat{\mathbf{x}}, \mathbf{x})$, it follows that $\operatorname{MSE}(\hat{\mathbf{x}}, p(\mathbf{x})) \geq \operatorname{MOSPA}(\hat{\mathbf{x}}, p(\mathbf{x}))=\operatorname{MOSPA}(\hat{\mathbf{x}})$. The notation $\operatorname{MOSPA}(\hat{\mathbf{x}})$ is introduced to stress that the evaluation of MOSPA is independent of the ordered density $p(\mathbf{x})$ (as long as it is in the same RFS family).

The following theorem shows an important relation between the MSE and the MOSPA.

Theorem 1 For any given $\hat{\mathbf{x}}$, there exists exactly one ordered density $\tilde{p}(\mathbf{x})$ within the RFS family, such that

$$
\operatorname{MSE}(\hat{\mathbf{x}}, \tilde{p}(\mathbf{x}))=\operatorname{MOSPA}(\hat{\mathbf{x}}) .
$$

Proof of Theorem 1 Let us define

$$
\tilde{p}(\mathbf{x})= \begin{cases}f\left(\left\{\mathbf{x}_{1}, \ldots, \mathbf{x}_{n}\right\}\right) & \text { if } \mathbf{x} \in \mathcal{A}(\hat{\mathbf{x}}) \\ 0 & \text { otherwise. }\end{cases}
$$

For this density $\tilde{p}(\mathbf{x})$, it now holds that

$$
\begin{aligned}
\operatorname{MSE}(\hat{\mathbf{x}}, \tilde{p}(\mathbf{x})) & =\int \frac{1}{n} \sum_{i=1}^{n} d\left(\hat{\mathbf{x}}_{i}, \mathbf{x}_{i}\right) \tilde{p}(\mathbf{x}) d \mathbf{x} \\
& =\int_{\mathbf{x} \in \mathcal{A}(\hat{\mathbf{x}})} \frac{1}{n} \sum_{i=1}^{n} d\left(\hat{\mathbf{x}}_{i}, \mathbf{x}_{i}\right) \tilde{p}(\mathbf{x}) d \mathbf{x} \\
& =\int_{\mathbf{x} \in \mathcal{A}(\hat{\mathbf{x}})} \tilde{d}_{p}^{(c)}(\hat{\mathbf{x}}, \mathbf{x}) \tilde{p}(\mathbf{x}) d \mathbf{x} \\
& =\operatorname{MOSPA}(\hat{\mathbf{x}}),
\end{aligned}
$$

In order to conclude the proof we need to show that the ordered density $\tilde{p}(\mathbf{x})$ is unique. Let us suppose that there exists a different ordered density $q(\mathbf{x})$ such that

$$
M S E(\hat{\mathbf{x}}, \tilde{p}(\mathbf{x}))-M S E(\hat{\mathbf{x}}, q(\mathbf{x}))=0 .
$$

Now, let us consider

$\operatorname{MSE}(\hat{\mathbf{x}}, \tilde{p}(\mathbf{x}))-\operatorname{MSE}(\hat{\mathbf{x}}, q(\mathbf{x}))$

$=\frac{1}{n} \int \sum_{i=1}^{n} d\left(\hat{\mathbf{x}}_{i}, \mathbf{x}_{i}\right)[\tilde{p}(\mathbf{x})-q(\mathbf{x})] d \mathbf{x}$

$=\frac{1}{n} \int_{\mathcal{A}(\hat{\mathbf{x}})} \sum_{i=1}^{n} d\left(\hat{\mathbf{x}}_{i}, \mathbf{x}_{i}\right)[f(\{\mathbf{x}\})-q(\mathbf{x})] d \mathbf{x}$

$-\frac{1}{n} \sum_{j=2}^{n !} \int_{\mathcal{A}(\hat{\mathbf{x}})} \sum_{i=1}^{n} d\left(\hat{\mathbf{x}}_{i}, \pi_{j}\left(\mathbf{x}_{i}\right)\right) q\left(\pi_{j}(\mathbf{x})\right) d \mathbf{x}$

$=\frac{1}{n} \int_{\mathcal{A}(\hat{\mathbf{x}})} \sum_{i=1}^{n} d\left(\hat{\mathbf{x}}_{i}, \mathbf{x}_{i}\right) \underbrace{\left[f(\{\mathbf{x}\})-q(\mathbf{x})-\sum_{j=2}^{n !} q\left(\pi_{j}(\mathbf{x})\right)\right]}_{=0} d \mathbf{x}$

$+\frac{1}{n} \sum_{j=2}^{n !} \int_{\mathcal{A}(\hat{\mathbf{x}})} \sum_{i=1}^{n}\left[d\left(\hat{\mathbf{x}}_{i}, \mathbf{x}_{i}\right)-d\left(\hat{\mathbf{x}}_{i}, \pi_{j}\left(\mathbf{x}_{i}\right)\right)\right] q\left(\pi_{j}(\mathbf{x})\right) d \mathbf{x}$

$=\frac{1}{n} \int_{\mathcal{A}(\hat{\mathbf{x}})} \sum_{j=2}^{n !} q\left(\pi_{j}(\mathbf{x})\right) \underbrace{\sum_{i=1}^{n}\left[d\left(\hat{\mathbf{x}}_{i}, \mathbf{x}_{i}\right)-d\left(\hat{\mathbf{x}}_{i}, \pi_{j}\left(\mathbf{x}_{i}\right)\right)\right]}_{<0 \quad \text { for each } j} d \mathbf{x}$

$=0$

$\Rightarrow \sum_{j=2}^{n !} q\left(\pi_{j}(\mathbf{x})\right)=0$

$\Rightarrow q(\mathbf{x})=\tilde{p}(\mathbf{x})$.

where $\pi_{j}\left(\mathbf{x}_{i}\right)$ denotes the change of the target index from $i$ to $j$. For instance, when $n=2, \pi_{1}\left(\mathbf{x}_{1}\right)=\mathbf{x}_{1}$, $\pi_{1}\left(\mathbf{x}_{2}\right)=\mathbf{x}_{2}, \pi_{2}\left(\mathbf{x}_{1}\right)=\mathbf{x}_{2}$ and $\pi_{2}\left(\mathbf{x}_{2}\right)=\mathbf{x}_{2}$.

The main result of the paper is described by the next theorem.

Theorem 2 The expression of the MMOSPA-estimate $\hat{\mathbf{x}}^{M M O S P A}$ is given by

$$
\hat{\mathbf{x}}^{M M O S P A}=E_{\tilde{p}(\mathbf{x})}\{\mathbf{x}\} .
$$

Proof of Theorem $\mathbf{2}$ To show that it leads to a contradiction, suppose $\check{\mathbf{x}} \neq \hat{\mathbf{x}}$ where $\check{\mathbf{x}}=E_{\tilde{p}(\mathbf{x})}\{\mathbf{x}\}$. As always, $\check{\mathbf{x}}=E_{\tilde{p}(\mathbf{x})}\{\mathbf{x}\}$ is the MMSE estimate, i.e., it is the unique minimum to $M S E(\check{\mathbf{x}}, \tilde{p}(\mathbf{x}))$. The assumption that $\check{\mathbf{x}} \neq \hat{\mathbf{x}}$ therefore gives that

$$
\operatorname{MSE}(\check{\mathbf{x}}, \tilde{p}(\mathbf{x}))<\operatorname{MSE}(\hat{\mathbf{x}}, \tilde{p}(\mathbf{x}))=\operatorname{MOSPA}(\hat{\mathbf{x}}) .
$$

As a consequence of (15) and from the fact that $\operatorname{MSE}(\hat{\mathbf{x}}, \tilde{p}(\mathbf{x})) \geq \operatorname{MOSPA}(\hat{\mathbf{x}})$, it follows that $\operatorname{MOSPA}(\check{\mathbf{x}}) \leq \operatorname{MSE}(\check{\mathbf{x}}, \tilde{p}(\mathbf{x}))<\operatorname{MSE}(\hat{\mathbf{x}}, \tilde{p}(\mathbf{x}))=$ $\operatorname{MOSPA}(\hat{\mathbf{x}})$. This is clearly a contradiction, since $\hat{\mathbf{x}}$ is assumed to have optimal MOSPA performance. Hence, we must have $\hat{\mathbf{x}}^{M M O S P A}=E_{\tilde{p}(\mathbf{x})}\{\mathbf{x}\}$.

Theorem 2 states an important analogy between the MMSE estimator and the MMOSPA estimator: 
the MMOSPA estimator of the target states is still expressed as the conditional expectation of the target states, but the integration is carried out with respect to a special ordered density $\tilde{p}(\mathbf{x})$. An important consequence is that the problem of minimizing MOSPA is equivalent ${ }^{3}$ to the problem of minimizing the sum of the trace of the posterior covariances, that is $\frac{1}{n} \sum_{t=1}^{n} \operatorname{tr}\left\{\mathbf{P}^{t}\right\}$, where $\mathbf{P}^{t}$ is the posterior covariance of target $t$ given the density $\tilde{p}(\mathbf{x})$. This result shows that the switching criterion used in the SJPDA algorithm [10], is optimal in the MOSPA sense.

The following example illustrates the importance of the density $\tilde{p}(\mathbf{x})$ and its impact on the MMOSPA estimates.

Example 1 Suppose that $x_{1}$ and $x_{2}$ are independently distributed states of target 1 and target 2 such that

$$
\begin{aligned}
& x_{1} \sim \mathcal{N}\left(x_{1} ; 0,1\right) \\
& x_{2} \sim \mathcal{N}\left(x_{2} ; 1,1\right)
\end{aligned}
$$

where $\mathcal{N}(x ; \mu, \sigma)$ denotes the Gaussian distribution with mean $\mu$ and standard deviation $\sigma$. In Figure 2 the joint distribution of the targets state is depicted and it easily follows from the definition of MMSE that

$$
\begin{aligned}
& \hat{x}_{1}^{M M S E}=\mathbb{E}_{p(\mathbf{X})}\left\{x_{1}\right\}=0 \\
& \hat{x}_{2}^{M M S E}=\mathbb{E}_{p(\mathbf{X})}\left\{x_{2}\right\}=1
\end{aligned}
$$

where the density $p(\mathbf{x})=\frac{1}{\sqrt{2 \pi}} e^{-\frac{x_{1}^{2}}{2}} \frac{1}{\sqrt{2 \pi}} e^{-\frac{\left(x_{2}-1\right)^{2}}{2}}$. The MMOSPA estimates are computed using equation (14) where the density $\tilde{p}(\mathbf{x})$ is depicted in Figure 3. We have that

$$
\hat{\mathbf{x}}^{M M O S P A}=\mathbb{E}_{\tilde{p}(\mathbf{X})}\{\mathbf{x}\}=\left(\begin{array}{c}
-0.1996 \\
1.1996
\end{array}\right)
$$

We note that the MMOSPA estimates $\hat{\mathbf{x}}^{M M O S P A}$ are unlabeled, revealing the fact that the estimator provides a lower error between the joint estimate and the joint target (target 1 and target 2), at the price of losing the identities of the same estimates.

\subsection{A General MMOSPA Algorithm}

Based on Theorem 2, we propose an iterative optimization algorithm.

1. Initiate with $i=1$ and let $\hat{\mathbf{x}}_{0}^{M M O S P A}=E_{p_{0}(\mathbf{x})}\{\mathbf{x}\}$ where $p_{0}(\mathbf{x})$ represents an ordered density within the RFS; for instance $\hat{\mathbf{x}}_{0}^{M M O S P A}$ could be the MMSE estimates.

${ }^{3}$ The following result

$$
\operatorname{MSE}(\hat{\mathbf{x}}, \tilde{p}(\mathbf{x}))=\frac{1}{n} \sum_{t=1}^{n} \operatorname{tr}\left\{\mathbf{P}^{t}\right\} .
$$

follows from the definition of $\mathbf{P}^{t}$, [5].

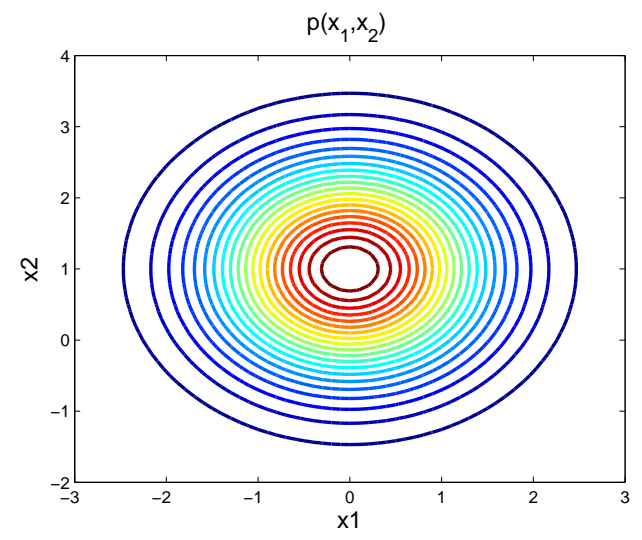

Figure 2: Joint distribution of the target states corresponding to a particular ordered density.

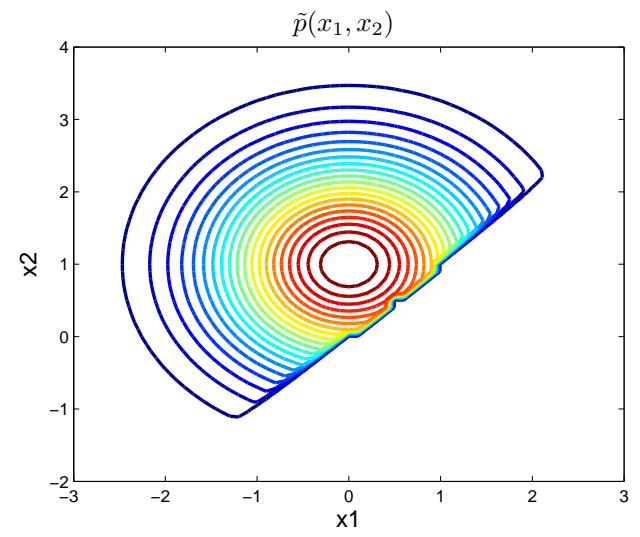

Figure 3: Density $\tilde{p}(\mathbf{x})$ given by the equation (11). 
2. Set $\mathcal{A}\left(\hat{\mathbf{x}}_{i}^{M M O S P A}\right)$ using the following equation

$$
\begin{aligned}
& \mathcal{A}\left(\hat{\mathbf{x}}_{i}^{M M O S P A}\right)=\left\{\mathbf{x}: \frac{1}{n} \sum_{j=1}^{n} d\left(\hat{\mathbf{x}}_{i-1, j}^{M M O S P A}, \mathbf{x}_{i}\right)=\right. \\
& \left.\tilde{d}_{p}^{(c)}\left(\hat{\mathbf{x}}_{i-1, j}^{M M O S P A}, \mathbf{x}\right)\right\},
\end{aligned}
$$

3. Select $\tilde{p}_{i}(\mathbf{x})$ as

$$
\tilde{p}_{i}(\mathbf{x})= \begin{cases}f\left(\left\{\mathbf{x}_{1}, \ldots, \mathbf{x}_{n}\right\}\right) & \text { if } \mathbf{x} \in \mathcal{A}\left(\hat{\mathbf{x}}_{i}^{M M O S P A}\right) \\ 0 & \text { otherwise. }\end{cases}
$$

4. Compute

$$
\hat{\mathbf{x}}_{i}^{M M O S P A}=E_{\tilde{p}_{i}(\mathbf{x})}\{\mathbf{x}\} .
$$

5. If $\hat{\mathbf{x}}_{i}^{M M O S P A} \approx \hat{\mathbf{x}}_{i-1}^{M M O S P A}$ we stop. Otherwise, set $i=i+1$ and go back to 2 .

The algorithm is essentially straightforward. However to implement it we need the ability to calculate the expected values with respect to the densities $\tilde{p}_{i}(\mathbf{x})$ as in equation (22). It appears complicated to find analytical expressions for those expected values in the general case. In the following subsection we presents an analytical expression for the MMOSPA estimates in the two-Gaussian-target case. In future work we intend to study the convergence of the iterative optimization algorithm and to suggest some numerical approximations for the computation of the MMOSPA estimates in the general case.

\subsection{Two Gaussian targets}

It appears difficult to find analytical expressions for the MMOSPA expression, given by Eq. (14), for any choice of the joint density $\tilde{p}(\mathbf{x})$. However, in most multi-target tracking applications, the posterior density is described as a weighted sum of Gaussian densities [5]. In this section we provide an analytical solution for $\hat{\mathbf{x}}^{M M O S P A}$ for the two-target case when the targets are independent and Gaussian. More specifically, let $\mathbf{x}=\left(\begin{array}{l}\mathbf{x}_{1} \\ \mathbf{x}_{2}\end{array}\right)$ and $\hat{\mathbf{x}}=\left(\begin{array}{l}\hat{\mathbf{x}}_{1} \\ \hat{\mathbf{x}}_{2}\end{array}\right)$ be the states and the estimates of the two targets, respectively. The dimensionality of targets state is $m$, that is $\mathbf{x}_{j}^{T}=\left(x_{j 1}, \ldots, x_{j m}\right)$, for $j=1,2$. For the two target case, the expression for $\mathcal{A}(\hat{\mathbf{x}})$ reduces to:

$$
\begin{aligned}
\mathcal{A}(\hat{\mathbf{x}}) & =\left\{\mathbf{x}: \frac{1}{2} \sum_{i=1}^{2} d\left(\hat{\mathbf{x}}_{i}, \mathbf{x}_{i}\right)=\overline{d_{p}^{(c)}}(\hat{\mathbf{x}}, \mathbf{x})\right\} \\
& =\left\{\mathbf{x}:\left(\hat{\mathbf{x}}_{2}-\hat{\mathbf{x}}_{1}\right)^{T}\left(\mathbf{x}_{1}-\mathbf{x}_{2}\right) \leq 0\right\} .
\end{aligned}
$$

Let us also introduce the matrix $\mathbf{B}$ :

$$
\mathbf{B}=\left(\begin{array}{cc}
-\mathbf{I}_{m} & \mathbf{I}_{m} \\
\mathbf{I}_{m} & -\mathbf{I}_{m}
\end{array}\right)
$$

where we denote with $\mathbf{I}_{m}$ the identity matrix with dimension $m \times m$. We can rewrite $\mathcal{A}(\hat{\mathbf{x}})$ in matrix form as follows:

$$
\mathcal{A}(\hat{\mathbf{x}})=\left\{\mathbf{x}: \hat{\mathbf{x}}^{T} \mathbf{B} \mathbf{x} \leq 0\right\}
$$

For the two target case we have that the RFS density can be represented by:

$$
f\left(\left\{\mathbf{x}_{1}, \mathbf{x}_{2}\right\}\right)=\mathcal{N}\left(\mathbf{x} ; \boldsymbol{\mu}_{12}, \mathbf{P}_{12}\right)+\mathcal{N}\left(\mathbf{x} ; \boldsymbol{\mu}_{21}, \mathbf{P}_{21}\right)
$$

where

$$
\begin{aligned}
& \boldsymbol{\mu}_{12}=\left(\begin{array}{l}
\boldsymbol{\mu}_{1} \\
\boldsymbol{\mu}_{2}
\end{array}\right), \boldsymbol{\mu}_{21}=\left(\begin{array}{l}
\boldsymbol{\mu}_{2} \\
\boldsymbol{\mu}_{1}
\end{array}\right) \\
& \mathbf{P}_{12}=\left(\begin{array}{lr}
\mathbf{P}_{1} & 0 \\
0 & \mathbf{P}_{2}
\end{array}\right) \mathbf{P}_{21}=\left(\begin{array}{lr}
\mathbf{P}_{2} & 0 \\
0 & \mathbf{P}_{1}
\end{array}\right),
\end{aligned}
$$

and where $\hat{\mathbf{x}}_{1}^{M M O S P A}$ can be written as:

$$
\begin{aligned}
& \hat{\mathbf{x}}_{1}^{M M O S P A}=E_{\tilde{p}_{(1)}(\mathbf{x})}\{\mathbf{x}\} \\
& =\int_{\hat{\mathbf{x}}_{0}^{M M O S P A T} \mathbf{B} \mathbf{x} \leq 0} \mathbf{x}\left[\mathcal{N}\left(\mathbf{x} ; \boldsymbol{\mu}_{12}, \mathbf{P}_{12}\right)\right. \\
& \left.+\mathcal{N}\left(\mathbf{x} ; \boldsymbol{\mu}_{21}, \mathbf{P}_{21}\right)\right] d \mathbf{x}
\end{aligned}
$$

The evaluation of equation (27) requires the derivation of the following general integral:

$$
\int_{\hat{\mathbf{x}}^{T} \mathbf{B} \mathbf{x} \leq 0} \mathbf{x} \mathcal{N}(\mathbf{x} ; \boldsymbol{\mu}, \mathbf{P}) d \mathbf{x}
$$

The expression of the closed form solution of the above integral is derived in the appendix.

\section{Evaluations}

In this section we validate our theoretical findings using an example. Consider a scenario where we have two Gaussian independently distributed targets; the probability of detection is one for both targets $(\mathrm{Pd}=1)$, and we have received two detections (here we assume the absence of clutter). The prior information about the target locations is given by the following densities:

$$
\begin{aligned}
& p\left(x_{1}\right)=\mathcal{N}\left(x_{1} ; 0,1\right) \\
& p\left(x_{2}\right)=\mathcal{N}\left(x_{2} ; 1,1\right)
\end{aligned}
$$

After collecting the two measurements we are not able to solve the ambiguity of their origin (measurement origin uncertainty), that is, to know which measurement comes from which target. We assume a Gaussian model for the measurement, that is

$$
\mathbf{z}=\mathbf{x}+\mathbf{w}
$$

with $w \sim \mathcal{N}\left(w ; 0, \sigma_{w}\right)$. Now we can write down the joint target density conditioned on the measurements as a weighted sum of Gaussian densities where each weight represents a particular global data association 


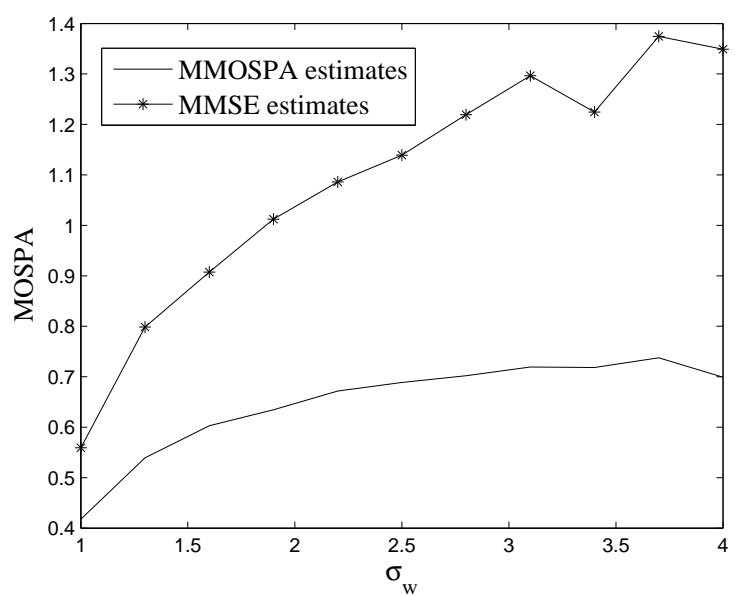

Figure 4: MOSPA performance of the MMOSPA estimator vs MMSE estimator. Here we used 1000 Monte Carlo trials.

probability [5]. Such a Gaussian mixture represents one particular ordered density within the RFS family

$$
\begin{aligned}
& p\left(x_{1}, x_{2} \mid \mathbf{z}\right)=P\left(H_{1}\right) p\left(x_{1}, x_{2} \mid H_{1} ; \mathbf{z}\right) \\
& +P\left(H_{2}\right) p\left(x_{1}, x_{2} \mid H_{2} ; \mathbf{z}\right)
\end{aligned}
$$

where $P\left(H_{1}\right)$ and $P\left(H_{2}\right)$ are the global data association probabilities and $p\left(x_{1}, x_{2} \mid H_{i} ; \mathbf{z}\right)$ is the joint Gaussian density. The MMSE estimates is then computed in the following way:

$$
\begin{aligned}
& \hat{x}_{1}^{M M S E}=\mathbb{E}_{p\left(x_{1}, x_{2} \mid \mathbf{z}\right)}\left\{x_{1}\right\} \\
& \hat{x}_{2}^{M M S E}=\mathbb{E}_{p\left(x_{1}, x_{2} \mid \mathbf{z}\right)}\left\{x_{2}\right\}
\end{aligned}
$$

whereas the MMOSPA estimates are expressed as

$$
\hat{\mathbf{x}}^{M M O S P A}=\mathbb{E}_{\tilde{p}\left(x_{1}, x_{2} \mid \mathbf{z}\right)}\{\mathbf{x}\}
$$

and where the above expectation can be computed using the closed form solution derived in the previous subsection. In Figure 4, the MOSPA for the MMOSPA estimator and the MMSE estimator are plotted versus the measurement noise. Clearly the MMOSPA estimator outperforms the MMSE estimator. A more interesting result is visualized in Figure 5, where the "kill probability" for the two targets is plotted versus the measurement noise for the MMOSPA and MMSE estimators, respectively. The kill probability is defined as the probability that the estimates of the target locations are within a ball of radius $\epsilon$ centered around the true target locations

$$
P_{k i l l}(\hat{\mathbf{x}}, \epsilon)=P(\|\hat{\mathbf{x}}-\mathbf{x}\| \leq \epsilon)
$$

This metric is very useful in the context of Anti-Ballistic Missiles (ABM) systems where the targets are the enemy missiles and the goal of the countermeasure systems is to try protect, without concern for which missile is the threat. As we can see from Figure 5, the

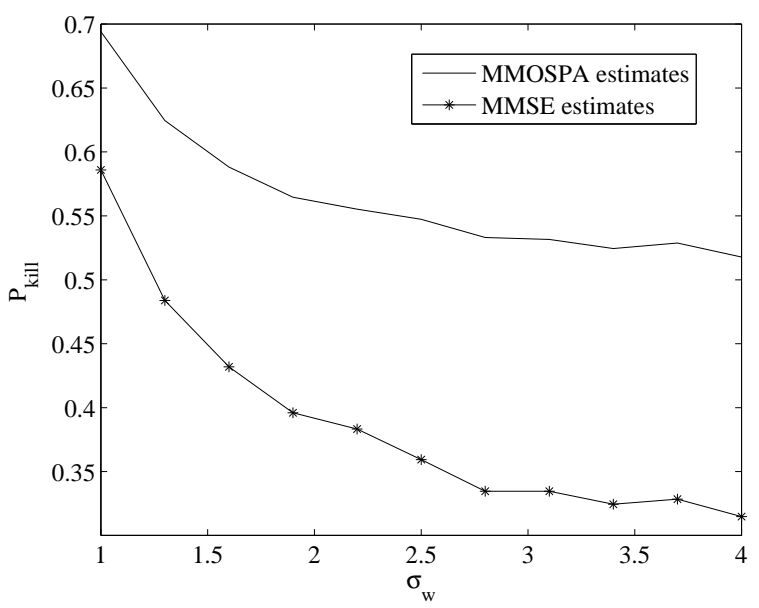

Figure 5: Kill probability $P_{\text {kill }}(\hat{\mathbf{x}}, \epsilon)$ for the MMOSPA and the MMSE estimators, respectively. Here we used 1000 Monte Carlo trials and $\epsilon=1$.

use of the MMOSPA estimator increases the chance to destroy both the targets.

\section{Conclusions}

Motivated by an interest to estimate a set of objects without regard to labeling, we have developed a formulation of the standard MMSE estimation problem that is label-free: a solution that reports that target 2 is close to the location of target 1 and vice versa ${ }^{4}$ is not penalized relative to one that correctly labels the objects. We derived a general algorithm which provides the MMOSPA estimates for an arbitrary, but known, number of targets. Estimation in this framework amounts to minimization with fewer constraints, and it is thence perhaps unsurprising that improved performance is possible even adopting standard metrics such as (unlabeled) MSE. What perhaps *is* surprising is the magnitude of the improvement.

\section{A Appendix}

Here we want to find a closed form solution of following integral:

$$
\int_{\hat{\mathbf{x}}^{T} \mathbf{B} \mathbf{x} \leq 0} \mathbf{x} \mathcal{N}(\mathbf{x} ; \boldsymbol{\mu}, \mathbf{P}) d \mathbf{x}
$$

Changing variable inside the integral, that is $\mathbf{y}=$ $\boldsymbol{\Lambda}^{-1 / 2} \mathbf{U}^{T}(\mathbf{x}-\boldsymbol{\mu})$, we have that:

$$
\begin{aligned}
& \int_{\hat{\mathbf{x}}^{T} \mathbf{B} \mathbf{x} \leq 0} \mathbf{x} \mathcal{N}(\mathbf{x} ; \boldsymbol{\mu}, \mathbf{P}) d \mathbf{x} \\
& =\int_{\hat{\mathbf{x}}^{T} \mathbf{B} \mathbf{U} \boldsymbol{\Lambda}^{1 / 2} \mathbf{y}+\hat{\mathbf{x}}^{T} \mathbf{B} \boldsymbol{\mu} \leq 0}\left(\mathbf{U} \boldsymbol{\Lambda}^{1 / 2} \mathbf{y}+\boldsymbol{\mu}\right) \times \mathcal{N}(\mathbf{y} ; \mathbf{0}, \mathbf{I}) d \mathbf{y}
\end{aligned}
$$

\footnotetext{
${ }^{4}$ Actually in our formulation there is no notion of "target 1 " at all.
} 
where $\mathbf{U}$ is the rotation matrix whose columns are the eigenvectors which are obtained by the eigendecomposition of $\mathbf{P}$ and $\boldsymbol{\Lambda}$ is the diagonal matrix of the eigenvalues, that is $\mathbf{P}=\mathbf{U} \boldsymbol{\Lambda} \mathbf{U}^{T}$. Let us define $\mathbf{a}^{T}=\hat{\mathbf{x}}^{T} \mathbf{B} \mathbf{U} \Lambda^{1 / 2}$ and $b=-\hat{\mathbf{x}}^{T} \mathbf{B} \boldsymbol{\mu}$. We now introduce a new change of variable $\mathbf{z}=\mathbf{A y}$, with $\mathbf{A}$ being an orthogonal matrix whose first row is equal to $\frac{\mathbf{a}^{T}}{\|\mathbf{a}\|}$ and the remaining orthogonal to each other. Thus, we have

$$
\begin{aligned}
& \int_{\hat{\mathbf{x}}^{T} \mathbf{B} \mathbf{U} \Lambda^{1 / 2} \mathbf{y}+\hat{\mathbf{x}}^{T} \mathbf{B} \boldsymbol{\mu} \leq 0}\left(\mathbf{U} \boldsymbol{\Lambda}^{1 / 2} \mathbf{y}+\boldsymbol{\mu}\right) \mathcal{N}(\mathbf{y} ; \mathbf{0}, \mathbf{I}) d \mathbf{y} \\
& =\int_{\mathbf{a}^{T} \mathbf{A}^{T} \mathbf{z} \leq b}\left(\mathbf{U} \boldsymbol{\Lambda}^{1 / 2} \mathbf{A}^{T} \mathbf{z}+\boldsymbol{\mu}\right) \mathcal{N}(\mathbf{z} ; \mathbf{0}, \mathbf{I}) d \mathbf{z} \\
& =\mathbf{U} \boldsymbol{\Lambda}^{1 / 2} \frac{\mathbf{a}}{\|\mathbf{a}\|} \int_{-\infty}^{\frac{b}{\|\mathbf{a}\|}} \tau \mathcal{N}(\tau ; 0,1) d \tau+\boldsymbol{\mu} \int_{-\infty}^{\frac{b}{\|\mathbf{a}\|}} \mathcal{N}(\tau ; 0,1) d \tau \\
& =-\mathbf{U} \boldsymbol{\Lambda}^{1 / 2} \frac{\mathbf{a}}{\|\mathbf{a}\|} \frac{e^{-\frac{b^{2}}{2\|\mathbf{a}\|^{2}}}}{\sqrt{2 \pi}}+\frac{\boldsymbol{\mu}}{2}\left(1+\operatorname{erf}\left(\frac{b}{\|\mathbf{a}\| \sqrt{2}}\right)\right)
\end{aligned}
$$

where $\operatorname{erf}(t)$ is the error function defined by $\operatorname{erf}(t)=\frac{2}{\sqrt{\pi}} \int_{0}^{t} e^{-\tau^{2}} d \tau$.

Thus, we have:

$$
\begin{aligned}
& \int_{\hat{\mathbf{x}}^{T} \mathbf{B} \mathbf{x} \leq 0} \mathbf{x} \mathcal{N}(\mathbf{x} ; \boldsymbol{\mu}, \mathbf{P}) d \mathbf{x} \\
& =-\mathbf{U} \boldsymbol{\Lambda}^{1 / 2} \frac{\mathbf{a}}{\|\mathbf{a}\|} \frac{e^{-\frac{b^{2}}{2\|\mathbf{a}\|^{2}}}}{\sqrt{2 \pi}}+\frac{\boldsymbol{\mu}}{2}\left(1+\operatorname{erf}\left(\frac{b}{\|\mathbf{a}\| \sqrt{2}}\right)\right)
\end{aligned}
$$

\section{References}

[1] O.E. Drummond and B.E. Fridling, "Ambiguities in evaluating performance of multiple target tracking algorithms," Signal and Data Processing of Small Targets, Proc. SPIE, pp. 326-337.

[2] J.R. Hoffman and R.P.S. Mahler, "Multitarget Miss Distance via Optimal Assignment," IEEE Transactions on Systems, Man, and Cybernetics - Part A: Systems and Humans, Vol 34, No. 3, pp. 327-336, May, 2000.

[3] D. Schuhmacher and B.-T. Vo and B.-N. Vo, "A Consistent Metric for Performance Evaluation of Multi-Object Filters," IEEE Transactions on Signal Processing, Vol 56, No. 8, pp. 3447-3457, August, 2008.

[4] R.P.S. Mahler, "Statistical Multisource-Multitarget Information Fusion," Artech House, 2007.

[5] Y. Bar-Shalom, and X. Li, Multitarget-Multisensor Tracking: Principles and Techniques, Storrs, CT: YBS Publishers, 1995.
[6] R.P.S. Mahler, "Multitarget Bayes Filtering via First-Order Multitarget Moments," IEEE Transactions on Aerospace and Electronic Systems, Vol 39, No. 4, pp. 1152-1178, October, 2003.

[7] R.P.S. Mahler, "PHD Filters of Higher Order in Target Number," IEEE Transactions on Aerospace and Electronic Systems, Vol 43, No. 4, pp. 15231543, October, 2007.

[8] B.-T. Vo and B.-N. Vo and A. Cantoni, "Analytic Implementations of the Cardinalized Probability Hypothesis Density Filter," IEEE Transactions on Signal Processing, Vol 55, No. 7, pp. 3553-3567, July, 2007.

[9] B-N. Vo and W.-K. Ma, "The Gaussian Mixture Probability Hypothesis Density Filter," IEEE Transactions on Signal Processing, Vol 54, No. 11, pp. 4091-4104, November, 2006.

[10] L. Svensson and D. Svensson and P. Willett, "Set JPDA algorithm for tracking unordered sets of targets," Proceedings of the 12th International Conference on Information Fusion, Seattle, USA, July, 2009 . 